\title{
Impact of PCI Timing on Short Term Outcome in Non ST Elevation Myocardial Infraction
}

\author{
Sayed Abd El.Khalik, Ali I. Atia, Hazem Abdelraouf, Al-Shimaa M. Sabry
}

\begin{abstract}
:
Department of cardiology, faculty of medicine, Benha University, Egypt.

Correspondence to: Background: Patients with non-ST-segment elevation myocardial infraction are at risk of adverse cardiac events like death, $\mathrm{CHF}$, reinfarction, stroke, major bleeding, cardiogenic shock, acute pulmonary Hazem Abdelraouf, Department of cardiology, faculty of medicine, Benha University, Egypt

Email:

h_raouf_92@yahoo.com

Received:13 November 2020

Accepted: 21 January 2021

edema and arrythmias. We aimed to investigate the outcome of early vs. late intervention in patients with non-ST-elevation myocardial infraction. Aim: To investigate the outcome of early vs. late intervention in patients with non-ST-elevation myocardial infraction. Subjects and methods: 100 eligible NSTEMI patients were included in our study and were divided into 2 groups: Group I "Early Intervention" 50 patients with door-to-balloon time $<24$ hours and Group II "Late Intervention" 50 patients with door-to-balloon time $>24$ hours. All patients were assessed for baseline characteristics, admission data, ECG, echocardiography, angiographic data in-hospital outcome \& 3 months follow up outcome. Results: An earlyintervention strategy did not differ from a delayed-intervention strategy in preventing a composite outcome of death, myocardial infarction, heart failure, cardiogenic shock, acute pulmonary edema, major bleeding stroke and arrythmias. Conclusion: Early invasive approach compared with a delayed invasive approach in patients with Non-STEMI does not improve survival.
\end{abstract}

Key words: Non-STEMI, PCI, Outcome.

\section{Introduction}

In contrast to St-segment elevation myocardial infarction (STEMI), where immediate coronary revascularization by percutaneous coronary intervention (PCI) for completely-occluded infarct-related artery is a guideline-mandated treatment, in non-ST-segment elevation myocardial infarction (NSTEMI) the optimal timing of 
coronary intervention is less clear as within NSTEMI there is non-occlusive plaque rupture. (1)

Over the past decade, two distinct approaches have emerged in the management of patients with non-ST elevation myocardial infraction (NSTEMI): (1) a routine early invasive strategy that consists of routinely referring patients with NSTEMI for coronary angiography during the first day after admission and if necessary, revascularization as soon as possible thereafter; and (2) a conservative strategy (also referred to as a selective invasive or ischemic-guided strategy) In this strategy, patients are initially treated with pharmacological therapy, after which coronary angiography and revascularization, if appropriate, are performed. (2)

We aimed to investigate the outcome of early vs. late intervention strategy in patients with non-ST-elevation myocardial infraction

\section{Patient and Method}

\section{Patients:}

This was a single centre prospective study that was conducted at Nation
Heart Institute from November 2019 to May 2020 and included 100 patients with NSTEMI. They were divided into 2 groups according to the time of intervention: "Early Intervention" 50 patients with door-to-balloon time $<24$ hours and "Late Intervention" 50 patients with door-to-balloon time $>24$ hours.

All the patients were followed up for 3 months from the date of admission.

Exclusion criteria were: ST-segment elevation myocardial infraction, unstable angina, active bleeding, any contraindication for the use of dual antiplatelet therapy, and presence of comorbidities with a life expectancy $<6$ months.

All Patients provided informed consent to participate in this study. The study was approved by our hospital ethics committee

Echocardiography:

Transthoracic echocardiography was performed within the 12 hours from presentation then at follow up (3months) to assess LV ejection fraction with Siemens ACUSON X700 ultrasound system.

Left ventricular end systolic volume (ESV), end-diastolic volume (EDV) and 
LV ejection fraction (EF) were measured from apical two and four chamber views using modified Simpson's method.

The left ventricle was divided into 17 segments to calculate WMSI as the sum of segments scores divided by the number of segments. Segments were scored as 1 , normal; 2, hypokinesia; 3, akinesia; 4, dyskinesia. (3)

Coronary angiography:

It was performed according to standard rules followed by PCI.

\section{Outcome:}

Hospital and three months follow up for (death, CHF, re-infarction, stroke, major bleeding, cardiogenic shock, acute pulmonary edema and arrythmias) were recorded

Cardiac remodeling was evaluated at three moths follow up and defined as $>20 \%$ increase in left ventricular end-diastolic volume (LVEDV).

\section{Statistical analysis}

Data were collected, revised, coded and entered to the Statistical Package for Social Science (IBM SPSS) version 20 ( Armonk, NY-USA: IBM Corp). Qualitative data were presented as number and percentages while quantitative data were presented as mean, standard deviations and ranges when parametric. The comparison between two groups with qualitative data were done by using Chi-square test and/or Fisher exact test was used instead of Chi-square test when the expected count in any cell was found less than 5 .

The comparison between two groups regarding quantitative data with parametric distribution was done by using Independent t-test. The confidence interval was set to 95\% and the margin of error accepted was set to $5 \%$. So, the p-value was considered significant as the following: Significance of results:

- Non-significant: $P$ value $>0.05$.

- Significant : $P$ value $<0.05$

- Highly significant : $P$ value $<0.001$

\section{Results}

There were no statistically significant differences between two groups regarding gender (the early intervention group included 34 males "68\%" and 16 females "32\%" while, the late intervention included 28 males "56\%" and 22 females "44\%", $\mathrm{P}=0.216)$ and age with mean age 53.6 +11.53 vs. $54+7.2$ years old, $\mathrm{P}=0.569$ ). 
There were no statistical significant differences between two groups regarding hypertension (29 patients "58\%" vs. 36 patients "72\%", $\mathrm{P}=0.142)$, DM (20 patients "40\%" vs. 29 patients "58\%", $\mathrm{P}=0.072$ ), smoking (27 patients "54\%" vs. 19 patients "38\%", $\mathrm{P}=0.108$ ), dyslipidemia (18 patients "36\%" vs. 19 patients "38\%", $\mathrm{P}=0.836)$ and family history of CAD (8 patients "16\%" Vs. 7 patients "14\%", $\mathrm{P}=0.841$ )

There were no statistical significant difference between two groups regarding systolic BP (127.46 \pm 22.7 vs. $123.4 \pm$ $16.98 \mathrm{mmHg}, \mathrm{P}=0.226)$, diastolic $\mathrm{BP}(80.2$ \pm 14.21 vs. $75.4 \pm 11.64 \mathrm{mmHg}, \mathrm{P}=0.068)$ and heart rate $(81.4 \pm 12.05$ vs. $78.6 \pm$ 11.07 bpm, $\mathrm{P}=0.238$ )

More patients in the early intervention group had ECG changes compared to the late intervention one (46 patients "92\%" vs. 33 patients "66\%", $\mathrm{P}=0.003$ ) ,but there was no significant statistical difference between two groups regarding type of change except for $\mathrm{T}$-wave inversion which is more in the early intervention group (32 patients "64\%" vs. 22 patients "44\%", P= $0.041)$

Troponin and CK MB levels were significant higher in the early intervention group $(1.8 \pm 2.1$ vs. $0.8 \pm 1.2 \mathrm{ng} / \mathrm{mL}$, $\mathrm{P}=0.043$ and $101.18 \pm 33.9$ vs. $83.68 \pm$ $30.49 \mathrm{U} / \mathrm{L}, \mathrm{P}=0.002$, respectively)

There were no statistically significant differences between the two studied groups regarding baseline LVEDV, LVESV and LVEF (131.6 \pm 38.52 vs. $136.94 \pm 44.03$ $\mathrm{ml}, \mathrm{P}=0.52,53.56 \pm 20.41$ vs. $65.8 \pm 37.18$ $\mathrm{ml}, \mathrm{P}=0.296,55.38 \pm 8.99$ vs. $55.16 \pm 9.75$ $\%, \quad \mathrm{P}=0.907$ respectively). Moreover, follow up values were not significantly different from baseline values. Single and two vessels were more found in late group, while three vessels were more in early group.

The early intervention group had 13 patients "26\%" with single vessel disease, 18 patients "36\%" with two vessels disease, and 19 patients "38\%" with three vessels disease. While the late intervention group had 20 patients "40\%" with single vessel disease, 20 patients "40\%" with two vessels disease, and 10 patients "20\%" with three vessels disease, $\mathrm{P}=0.043$ ).

There was no statistically significant difference between two groups regarding site of lesion. The early intervention group included 6 patients "12\%" with LM lesion, 41 patients "82\%" with LAD lesion, 35 patients "70\%" with LCX lesion and 28 
patients "56\%" with RCA lesion. While the late intervention group included 1 patient "2\%" with LM lesion, 34 patients "68\%" with LAD lesion (30 patients "60\%" with LCX lesion and 26 patients "52\%" with RCA lesion).

There was no statistical significant difference between the two groups regarding in hospital outcome. None of patients of both groups had in hospital mortality or stroke. There were no significant statistical difference between the two groups regarding re-infraction only 1 patient in the late intervention group, heart failure (10 patients "20\%" vs. 8 patients "16\%", $\mathrm{P}=0.603$ ), cardiogenic shock only 1 patient in the late intervention group, major bleeding only 1 patient in the early group, acute pulmonary edema (7 patients "14\%" vs. 5 patients "10\%", $\mathrm{P}=0.538$ ), arrythmias (4 patients "8\%" vs. 2 patients "4\%", $\mathrm{P}=0.678$ ).

There was no significant statistical difference between the two groups regarding Three months follow. None of patients of both groups reported cardiogenic shock, stroke, major bleeding, acute pulmonary edema or arrythmias). Only 1 patient died in early group during follow up period. There were no significant statistical difference between the two groups regarding re-infraction, (2 Patients "4\%" in both groups $\mathrm{P}>0.999)$, heart failure (4 patients "8\%" in both groups, $\mathrm{P}>0.999$ ) and LV remodeling (13 patients "26\%" vs. 10 patients "20\%",P=0.579).

Table (1) Comparison between the studied groups regarding in hospital outcome:

\begin{tabular}{lllll}
\hline & $\begin{array}{l}\text { Early Intervention } \\
\mathbf{N = 5 0}(\boldsymbol{\%})\end{array}$ & $\begin{array}{l}\text { Late Intervention } \\
\mathbf{N = 5 0}(\boldsymbol{\%})\end{array}$ & $\chi^{2} / \mathbf{p}$ & $\mathbf{p}$ \\
\hline Mortality: & $0(0)$ & $0(0)$ & Fisher & $>0.999$ \\
Reinfarction: & $0(0)$ & $1(2)$ & Fisher & $>0.999$ \\
Heart failure: & $10(20)$ & $8(16)$ & 0.271 & 0.603 \\
Cardiogenic shock: & $0(0)$ & $1(2)$ & Fisher & $>0.999$ \\
Stroke: & $0(0)$ & $0(0)$ & Fisher & $>0.999$ \\
Major bleeding: & $1(2)$ & $0(0)$ & Fisher & $>0.999$ \\
Acute Pulmonary Edema: & $7(14)$ & $5(10)$ & 0.379 & 0.538 \\
Arrhythmia: & $4(8)$ & $2(4)$ & Fisher & 0.678 \\
\hline
\end{tabular}


Table (2) : Comparison between the studied groups regarding three months follow-up:

\begin{tabular}{lllll}
\hline & $\begin{array}{l}\text { Early Intervention } \\
\mathbf{N = 5 0}(\boldsymbol{\%})\end{array}$ & $\begin{array}{l}\text { Late Intervention } \\
\mathbf{N = 5 0}(\mathbf{\%})\end{array}$ & $\chi^{2} \mathbf{p}$ & $\mathbf{p}$ \\
\hline Mortality: & $1(2)$ & $0(0)$ & Fisher & $>0.999$ \\
Reinfarction: & $2(4)$ & $2(4)$ & Fisher & $>0.999$ \\
Heart failure: & $4(8)$ & $4(8)$ & Fisher & $>0.999$ \\
Cardiogenic shock: & $0(0)$ & $0(0)$ & Fisher & $>0.999$ \\
Stroke: & $0(0)$ & $0(0)$ & Fisher & $>0.999$ \\
Major bleeding: & $0(0)$ & $0(0)$ & Fisher & $>0.999$ \\
Acute Pulmonary Edema: & $0(0)$ & $0(0)$ & Fisher & $>0.999$ \\
Arrhythmia: & $0(0)$ & $0(0)$ & Fisher & $>0.999$ \\
Remodeling: & $13(26)$ & $10(20)$ & Fisher & 0.579 \\
\hline
\end{tabular}

\section{Discussion}

The optimal timing of revascularization in patients with non-ST-segment elevation myocardial infarction (NSTEMI) continues to be rigorously debated. Early intervention has the potential to prevent ischemic events during the waiting time from event to revascularization (5). Conversely, a delayed intervention may avoid procedure-related complications by allowing plaque to stabilize during the waiting period, as the patient undergoes medical therapy (6). In ST-elevation myocardial infarction (STEMI), there is complete occlusion of the coronary artery resulting in a need for immediate transfer to the cardiac catheter lab for primary percutaneous coronary intervention (PCI) to achieve reperfusion of the myocardium and improve clinical outcomes (7). In contrast, non-ST elevation myocardial infarction (NSTEMI), can present in a heterogeneous manner. Patients may suffer chest discomfort or shortness of breath only, with biomarker evidence of myocardial injury or present with acute hemodynamic compromise. The variations in presentation make decisions around timing of intervention less categoric than with STEMI patients (8).

The aim of this study is to evaluate whether and how the timing of percutaneous coronary intervention (PCI) affects the 
short-term outcome in patients presenting with NSTEMI.

In this study we investigate the outcome of early vs. late intervention in patients with non-ST-elevation myocardial infraction presenting in National Heart Institute Cairo, Egypt. One hundred eligible randomly selected NSTEMI patients were included in our study from November 2019 to May 2020. They were divided into 2 groups:

"Early intervention" 50 patients with doorto-balloon time $<24$ hours and "Late intervention" 50 patients with door-toballoon time $>24$ hours. All the patients were followed up for 3 months from the date of admission.

This current study has no statistically significant difference between two groups regarding gender and age. This was similar with other studies which studied "Outcome of Early vs. Delayed Invasive Intervention in Acute Coronary Syndrome". It was found that there was no difference between two groups regarding age and gender (9).

This current study has no statistically significant difference between two groups regarding hypertension, DM, smoking and dyslipidemia. This was similar with other studies which studied the "Association between time to percutaneous coronary intervention and hospital mortality in nonSTEMI". It was found that no statistically significant difference between two groups regarding hypertension, DM, smoking and dyslipidemia (10).

This current study has no significant difference between two groups regarding systolic BP, diastolic BP and heart rate.

This current study has more patients in the early intervention group had ECG changes compared to the late intervention one $(92 \%$ vs. $66 \%$ ). This was different to other studies which studied "Early versus Delayed Invasive Intervention in Acute Coronary Syndromes" They found that there was no significant statistical difference between both groups regarding ECG changes $(80.5 \%$ Vs 79.9\%) (11). This may be due to the large number of patients (3031 patients) compared to current study (100 patients). But there was no significant statistical difference between two groups regarding type of ECG change except for T-wave inversion which is more in the early intervention group.

In current study troponin and CK-MB levels were significant higher in the early intervention group. This was similar to other studies done on the study question "Should non-ST-Elevation myocardial infarction be 
treated like ST-Elevation myocardial infarction with shorter door-to-balloon time?" They found that cardiac enzymes were higher in early group (12).

In current study there was no statistically significant difference between the two studied groups regarding baseline LVEDV, LVESV and LVEF. Moreover, follow up values were not significantly different from baseline values. This was similar to another study (13) done on "Comparison of immediate vs early invasive strategy in patients with first acute non-ST-elevation myocardial infarction". They found that there was no significant statistical difference between both groups in baseline and follow up LVEDV, LVESV and LVEF.

In current study single and two vessels were more in late group, and three vessels was more in early group. This was in contrast to (9) which tested "Outcome of Early vs. Delayed Invasive Intervention in Acute Coronary Syndrome". They found that single vessel disease was more in the early intervention while two and three vessels disease were more prevalent in the delayed intervention group. This could be explained by the presence of STEMI patients within early primary PCI in their study. In current study there was no significant difference between two groups regarding site of lesion.
This was similar to other studies like (14) which were done on "Timing of intervention in high-risk non-ST-elevation acute coronary syndromes in PCI versus non-PCI centers.

In current study there was no statistically significant difference between the two groups regarding in hospital outcome. None of patients of both groups had in hospital mortality or stroke. There was no significant statistical difference between the two groups regarding re-infraction, heart failure, cardiogenic shock, major bleeding, acute pulmonary edema, and arrhythmias. This was similar to other studies like (15) who studied "Immediate vs Delayed Intervention for Acute Coronary Syndromes". They found that there was no significant difference between two groups regarding in hospital outcome.

In current study there was no significant statistical difference between the two groups regarding three months follow up outcome. None of patients of both groups reported cardiogenic shock, stroke, major bleeding, acute pulmonary edema or arrythmias. Only 1 patient died in early group. There was no significant statistical difference between the two groups regarding re-infraction, heart failure and LV remodeling. This was similar to other studies like (16) which studied 
"two-year outcome after early or late intervention in non-ST elevation acute coronary syndrome. They found that there is no significant difference between the two groups regarding 30 days and two-year death, reinfarction and recurrent ischemia. Similarly, in a study (12) which was done to answer the question "Should non-STElevation myocardial infarction be treated like ST-Elevation myocardial infarction with shorter door-to-balloon time?" They found that there were no significant statistical differences between both groups in follow up outcome and LV remodeling.

\section{Conclusion}

Early invasive approach compared with a delayed invasive approach in patients with Non-STEMI does not improve survival.

\section{References}

1. Steg P. G., James S. K., Atar D., Badano L. P., Lundqvist C. B., Borger M. A., et al., (2012): ESC guidelines for the management of acute myocardial infarction in patients presenting with ST-segment elevation. Eur Heart J ;33 :2569-2619.

2. Amsterdam E. A., Wenger N. K., Brindis R. G., Casey D. E., Ganiats T. G., Holmes D. R., et al., (2014): 2014 AHA/ACC guideline for the management of patients with non-ST-elevation acute coronary syndromes: a report of the American College of Cardiology/American Heart Association Task Force on Practice
Guidelines. Journal of the American College of Cardiology; 64(24): e139-e228.

3. Lang R. M., Badano L. P., Mor-Avi V., Afilalo J., Armstrong A., Ernande L.,et al., (2015): Recommendations for cardiac chamber quantification by echocardiography in adults. European Heart Journal-Cardiovascular Imaging; 16(3): 233-271.

4. Cokkinos D. V. and Belogianneas C. (2016): Left ventricular remodeling: a problem in search of solutions. European Cardiology Review; 11(1): 29.

5. Neumann FJ, Kastrati A, Pogatsa-Murray G, Mehilli J, Bollwein H, Bestehorn HP., et al., (2003): Evaluation of prolonged antithrombotic pretreatment ("cooling-off" strategy) before intervention in patients with unstable coronary syndromes: a randomized controlled trial. JAM 290: $1593-1599$.

6. Riezebos R. K., Ronner E., Ter Bals E., Slagboom T., Smits P. C., Ten Berg J. M., et al., (2009): OPTIMA trial. Immediate versus deferred coronary angioplasty in non-STsegment elevation acute coronary syndromes. Heart; 95: $807-812$.

7. Windecker S., Kolh P., Alfonso F., Collet J. P., Cremer J., Falk V., et al., (2015): 2014 ESC/EACTS guidelines on myocardial revascularization. EuroIntervention; 10(9): 1024-1094.

8. Roffi M, Patrono C, Collet JP, Mueller C, Valgimigli M, Andreotti F., et al., (2016): 2015 ESC Guidelines for the management of acute coronary syndromes in patients presenting without persistent ST-segment elevation: Task Force for the Management of Acute Coronary Syndromes in Patients Presenting without Persistent ST-Segment Elevation of the 
European Society of Cardiology (ESC). European heart journal; 37(3): 267315.

9. Shafi, M., and Nasrin, S. (2019). Outcome of Early vs. Delayed Invasive Intervention in Acute Coronary Syndrome Patients Attending a Selected Specialized Hospital: A Comparative Study. Cardiovascular Journal, 11(2), 129-138.

10. Kim T. G., Do Shin S., Song K. J., Lee Y. J., Lee E. J., Ro Y. S., et al., (2015): Association between time to percutaneous coronary intervention and hospital mortality in nonSTEMI: a prospective multicenter observational study. The American journal of emergency medicine; 33(11): 1591-1596.

11. Mehta SR, Granger CB, Boden WE, Steg PG, Bassand JP, Faxon DP., et al (2009): Early versus delayed invasive intervention in acute coronary syndromes. New England Journal of Medicine; 360(21): 2165-2175.

12. Iantorno M., Shlofmitz E., Rogers T., Torguson R., Kolm P., Gajanana D., et al., (2020): Should Non-ST-Elevation Myocardial Infarction be Treated like ST-Elevation Myocardial Infarction With Shorter Door-to-Balloon Time? The American Journal of Cardiology; 125(2): 165-168.
13. Sciahbasi A., Madonna M., De Vita M., Agati L., Scioli R., Summaria F., et al., (2010): Comparison of Immediate vs Early Invasive Strategy in Patients with First Acute Non-STElevation Myocardial Infarction. Clinical cardiology, 33(10), 650-655.

14. Badings E. A., Remkes W. S., Dambrink J. E., Van Wijngaarden J., Tjeerdsma G., Rasoul S., et al (2016): Timing of intervention in high-risk non-ST-elevation acute coronary syndromes in PCI versus non-PCI centers. Netherlands Heart Journal; 24(3): 181-187.

15. Montalescot G., Cayla G., Collet J. P., Elhadad S., Beygui F., Le Breton H.,et al (2009): Immediate vs delayed intervention for acute coronary syndromes: a randomized clinical trial. Jama; 302(9): 947-954.

16. Badings E. A., Remkes W. S., Dambrink J. H. E., Tjeerdsma G., Rasoul S., Timmer J. R., et al., (2017): Two-year outcome after early or late Intervention in non-ST elevation acute coronary syndrome; Open heart: 4(1).

To cite this article: Sayed Abd El.Khalik , Ali I. Atia, Hazem Abdelraouf , Al-Shimaa M. Sabry. Impact of PCI Timing on Short Term Outcome in Non ST Elevation Myocardial Infraction BMFJ 2021; 38(2): 455-464, DOI: 10.21608/bmfj.2021.49722.1343 